\title{
METODOLOGÍAS ACTIVAS Y EVALUACIÓN FORMATIVA
}

Active methodologies and formative assessment

Metodologias ativas e avaliação formativa

Ángel Pérez-Pueyo

Universidad de León, España. Teléfono: +34 619867767. E-mail: angel.perez.pueyo@unileon.es

\begin{abstract}
Resumen
El trabajo en grupo se han convertido en una de las competencias transversales que más dificultades está generando en el desarrollo de un enfoque competencial del aprendizaje. El taller pretende lleva a cabo una experiencia a través del “Juego de Jack” en la que comprobar y sacar a la luz algunos de los problemas más habituales. Además, éste se complementará con algunos recursos e instrumentos que permitan al docente afrontar el desarrollo de la competencia desde una perspectiva más completa. La experiencia demuestra que identificando en primera persona los problemas que se plantean es más fácil afrontarlos con el alumnado.

Palabras clave: Trabajo en grupo; el Juego de Jack; instrumentos de evaluación
\end{abstract}

\begin{abstract}
Group work has become one of the transversal competences that is creating more difficulties in the development of a competential approach to learning. The workshop aims to carry out an experience through the "Game of Jack" in which to check and bring to light some of the most common problems. In addition, this will be complemented with some resources and instruments that allow the teacher to face the development of the competition from a more complete perspective. Experience shows that identifying in the first person any problems presented it is easier to deal with them with the students.

Keywords: Group work; The Jack Game; evaluation instruments
\end{abstract}

\section{Resumo}

O trabalho em grupo tornou-se uma das competências transversais que está gerando mais dificuldades no desenvolvimento de uma abordagem de competências para a 
aprendizagem. Os objectivos do workshop realiza uma experiência através da "Game Jack", que verificar e trazer à luz alguns dos problemas mais comuns. Além disso, este será complementado por alguns recursos e ferramentas para desenvolver os professores enfrentam a concorrência de uma perspectiva mais abrangente. A experiência mostra que a identificação em primeira mão os problemas encontrados é mais fácil enfrentá-los com os alunos.

Palavras-chave: Trabalho em grupo; Jogo Jack; ferramentas de avaliação

\section{Los instrumentos de evaluación: elemento clave en la evaluación formativa}

Los enfoques pedagógicos innovadores que hoy parecen enriquecer el contexto docente, en algunos casos no han surgido en la actualidad. La Escuela inclusiva de las Comunidades de aprendizaje (Ayuste, Flecha, López, \& Lleras, 1994), el Aprendizaje basado en proyectos y en problemas (Aranda, 2009; Bas, 2011), las Inteligencias múltiples (Gardner, 1983), el Aprendizaje cooperativo (Johnson, Johnson, \& Holubec, 1999; Pujolás, 2010), el Pensamiento visible (Del Pozo, 2012), o el Aprendizajeservicio (Martínez, 2008) son solo algunas de las propuestas que se unen a otras más clásicas que vuelven a recuperar auge como Waldorf (Moreno, 2010) o Montessori (Montessori \& de Sarmiento, 1979).

Si algo caracteriza a todas y cada una de las propuestas es que dejan de dar tanta importancia al trabajo individual para afrontar el trabajo grupal y el desarrollo de las capacidades de relaciones interpersonales, de inserción social y, por supuesto, las afectivo-motivacionales o de autonomía y equilibrio personal vinculadas a la autoestima.

Por eso, este taller se va a centrar en un ejemplo de cómo hacer reflexionar, tanto a docentes como al alumnado, sobre su influencia en el resultado y en el proceso de aprendizaje de todos. En cómo la educación de los individuos puede reorientar la actividad hacia situaciones inesperadas, y debemos contar con ello.

Y además, tras la evidencia científica que ha demostrado las enormes ventajas de la evaluación formativa para la mejora del aprendizaje del alumnado (Biggs, 2005; Boud, 1995; Boud \& Falchikov, 2007; López-Pastor, 1999, 2006, 2013), hemos incorporado la presentación de algunos instrumentos que complementen en proceso de 
aprendizaje de los aspectos sociales (López-Pastor, \& Pérez-Pueyo, 2017; Pérez-Pueyo, Hortigüela, \& Gutiérrez-García, 2017).

\section{Contextualización del taller: Todas las etapas educativas}

Tanto en el ámbito universitario como en el no universitario, el trabajo en grupo es una de las actividades más habituales y que, curiosamente, menos suele gustar al alumnado por la repercusión que suele tener en la calificación. De hecho, el desarrollo de esta competencia transversal tiene muchos aspectos a tener en cuenta y, según cómo se planteen o las características del alumnado y/o del docente, puede derivar en que se coopere, se colabore e incluso se compita; y en consecuencia, las estrategias y condiciones a tener en cuenta son muy diferentes.

La identificación y reparto de los diferentes roles que se pueden observar en la realización del mismo y la falta de equidad en la participación de sus componentes son algunos de los problemas fundamentales para conseguir que los miembros del grupo se encuentren cómodos trabajando. Además, se nos ha hecho creer que el objetivo del trabajo en grupo es que todos trabajen igual para que de ese modo todos puedan tener la misma nota.

Sin embargo, en vez de hacernos comprender que es imposible que todos los miembros trabajen igual (pero que conseguir que todos cumplan con el trabajo encomendado permite que se alcancen las metas esperadas), nos han hecho creer que la individualización (hacer el trabajo solo) o las situaciones de exclusión (no permitiendo que determinados compañeros participen en el grupo) son las únicas soluciones posibles.

En este sentido, la obsesión por la calificación tampoco permite valorar la posibilidad, utilizando las estrategias y recursos adecuados, de llegar a un acuerdo para que el valor del trabajo realizado o aportado sea satisfactorio para todos los miembros del grupo sin que sea necesario que todos tengan el mismo resultado.

En realidad, olvidamos que el objetivo debería ser que todos alcancen cotas similares de conocimiento y aprendizaje, aunque no necesariamente iguales, y que el reconocimiento del resultado individual sólo debería ser una consecuencia lógica del trabajo realizado (Pérez-Pueyo, 2005). 


\section{Descripción del taller}

El taller comienza con la realización de la actividad "El juego de Jack”1 (LIE, n.d). Este maravilloso juego nos permite, con independencia del contexto de docentes o alumnos en el que lo pongamos en práctica, demostrar las sensaciones que se pueden llegar a experimentar o provocar en función de nuestra capacidad para sacarle partido a la actividad. Como es bien sabido, las cosas no son ni buenas ni malas, las hacemos buenas o malas nosotros.

El juego plantea la resolución de un problema: ¿Cuánto tiempo, en horas, le llevará a Jack el viaje desde Piedrasluengas hasta Castañeda, si pasa sin parar por Valderrobles y Aguasturbias? Y éste proporciona 16 informaciones que se reparten entre los miembro del grupo, que pueden compartir oralmente pero sin enseñárselas a los demás. Algunos de los datos ofrecidos son necesarios para la resolución del problema, aunque otros quizás no. La mayor curiosidad la genera el hecho de que las unidades de medida no son las habituales.

Como es lógico, nuestra educación individualista permite (a los que sean competentes matemáticos) resolver el problema con facilidad; aunque para otros, la frustración puede hacer mella. Sin embargo, el problema no se plantea individualmente sino de forma colectiva y es cuando se evidencia nuestro nivel de competencia y el de las diferentes dimensiones que la componen. En algunos casos se comprobará que los grupos encuentran la solución, pero a través de la resolución individual de uno de los miembros competentes matemáticamente hablando o del que adopta la función de líder por autoaclamación; lo que nos permitirá reflexionar sobre si la experiencia se ha afrontado desde la cooperación, la colaboración o la competición y las consecuencias que tiene en el resto de los participantes.

Aunque la actividad presenta alguna propuesta para la evaluación, éstas no afrontan el verdadero problema de la valoración del trabajo individual, por lo que completaremos el taller con diferentes recursos y estrategias para el reparto de notas tras la valoración del trabajo realizado (Pérez-Pueyo, 2017). Además, se plantearán algunos recursos para que el alumnado, cuando trabaje en grupo de manera regular, pueda recopilar información diaria de la aportación de cada miembro (Casado, \& Pérez-Pueyo,

\footnotetext{
${ }^{1}$ Material elaborado por el Laboratorio de innovación educativa de la Cooperativa Jose Ramón Otero basado en Ainscow (2001). 
2014, 2015). Esta proporcionará la información necesaria para que los miembros puedan tener argumentos para el reparto. También presentaremos algunas estrategias de reparto de mayor a menor implicación emocional (Pérez-Pueyo, 2017).

\section{Reflexiones finales}

El taller espera ofrecer a los docentes una actividad muy interesante desde el punto de vista del análisis de la participación grupal de un trabajo grupal y la aportación y el aprendizaje final de cada participante. Además, se completará con una serie de recursos y estrategias tanto para el seguimiento intragrupal como para el reparto posterior del valor del trabajo realizado por cada miembro.

\section{Referencias}

Ainscow, M. (2001). Desarrollo de escuelas inclusivas: ideas, propuestas y experiencias para mejorar las instituciones escolares. Madrid: Narcea.

Aranda, S. R. (2009). Aprendizaje basado en proyectos. Revista Innovación Experiencias Educativas, 24, 1-6.

Ayuste, A.; Flecha, R.; López, F., \& Lleras, J. (1994). Planteamientos de la pedagogía crítica. Comunicar y transformar. Barcelona: Graó.

Bas, E. (2011). Aprendizaje basado en problemas. Cuadernos de pedagogía, (409), 4244.

Biggs, J. (2005). Calidad del aprendizaje universitario. Madrid: Narcea.

Boud, D. (1995). Enhancing Learning Through Self-Assessment. London: Routledge.

Boud, D. \& Falchikov, N. (2007). Rethinking Assessment in Higher Education. Learning for the long term. Oxon: Routledge.

Casado, O.M., \& Pérez-Pueyo, A, (2014). Diario de seguimiento intragrupal. Modelo A. Recuperado de

\section{https://drive.google.com/file/d/0B7xEdPlO4dj8TEZWSmZVdlhFZ2c/view}

Casado, O.M., \& Pérez-Pueyo, A, (2015). Diario de seguimiento intragrupal. Modelo B. Recuperado de https://drive.google.com/file/d/0B7xEdPlO4dj8Y2s1dTI0RGdYTWc/view 
Del Pozo, M. (2012). Aprendizaje inteligente. Secundaria en el colegio Monsterrat. Barcelona: Tekman Books.

Gardner, H. (1983). Inteligencias múltiples. Paidós: Barcelona.

Johnson, D.W., Johnson, R.T. y Holubec, E. (1999). El aprendizaje cooperativo en el aula. Paidós: Buenos Aires.

Laboratorio de innovación educativa (s.f.). El juego de Jack. Madrid: Cooperativa Jose Ramón Otero.

López-Pastor, V. M. (1999) Prácticas de evaluación en Educación Física: estudio de casos en Primaria, Secundaria y Formación del Profesorado. Valladolid: Universidad de Valladolid.

López-Pastor, V.M. (Coord.) (2006). La Evaluación en Educación Física. Revisión de los modelos tradicionales y planteamiento de una alternativa: la evaluación formativa y compartida. Buenos Aires: Miñó y Dávila

López-Pastor, V. M. (2013). Evaluación en Educación Física. Revisión internacional de la temática. Revista de Educación Física. Renovar la teoría y la práctica, 29(3), 4-13.

López-Pastor, V. M. \& Pérez-Pueyo, A. (Coords.) (2017). Evaluación formativa y compartida en educación: experiencias de éxito en todas las etapas educativas. León: Universidad de León. Recuperado de https://buleria.unileon.es/handle/10612/5999

Martínez, M. (2008). Aprendizaje servicio y responsabilidad social de las universidades. Barcelona: Octaedro.

Montessori, M., \& de Sarmiento, S. L. (1979). La educación para el desarrollo humano: comprendiendo a Montessori. México: Diana.

Moreno, M. M. (2010). Pedagogía Waldorf/Waldorf Education. Arteterapia, 5, 203209.

Pérez-Pueyo, A. (2005). Estudio del planteamiento actitudinal del área de Educación Física de la Educación Secundaria Obligatoria en la LOGSE: Una propuesta didáctica centrada en una metodología basada en actitudes. León: Universidad de León. 
Pérez-Pueyo, A. (2017). El estilo actitudinal como propuesta metodológica vinculada a la evaluación formativa. En V. M. López-Pastor \& Pérez-Pueyo (coords) Evaluación formativa y compartida en educación: experiencias de éxito en todas las etapas educativas (pp. 240-259). León: Universidad de León.

Pérez-Pueyo, A., Hortigüela, D., \& Gutiérrez-García, C. (2017). Reflexión sobre la evaluación en la formación inicial del profesorado en España. En búsqueda de la concordancia entre dos mundos. Revista Infancia, Educación y Aprendizaje, 2(2), 39-75.

Pujolás, P. (2010). Aprendizaje cooperativo y educación inclusiva: una forma práctica de aprender juntos alumnos diferentes. Vic: Universidad de VIC. 